\title{
Thermophotovoltaic Cells Based on Low-Bandgap Compounds
}

\author{
V.P.Khvostikov, V.D.Rumyantsev, O.A.Khvostikova, M.Z.Shvarts \\ P.Y.Gazaryan, S.V.Sorokina, N.A.Kaluzhniy, V.M.Andreev
}

Ioffe Physico-Technical Institute, 26 Polytechnicheskaya, St.Petersburg, 194021, Russia

\begin{abstract}
High efficiency TPV GaSb and Ge based cells fabricated by a non-toxic and inexpensive $\mathrm{Zn}$-diffusion technique have been developed. GaSb based cells optimised for operation with solar powered photon emitter allowed increasing the efficiencies up to $27-28 \%$ at black body temperature $>2000 \mathrm{~K}$ assuming $90 \%$ reflection of sub-bandgap photons. Combination of the MOCVD technique or LPE growth and $\mathrm{Zn}$ diffusion from the gas phase allows fabricating Ge photocells on the base of the $\mathrm{GaAs} / \mathrm{Ge}$ heterostructures, which are characterized by high photocurrent and open circuit voltage values. Efficiencies of $13 \%$ were obtained in GaAs/Ge TPV cells under the black-body $(1700-2100 \mathrm{~K})$ irradiation assuming the achieved $90 \%$ reflection of sub-bandgap photons.
\end{abstract}

\section{INTRODUCTION}

Sunlight is an ecologically safety and available energy source with a high energetic potential. Employment of concentrated sunlight as a heat source in a TPV system [1-4] is promising for increase in TPV conversion efficiency with retention of all advantages of sunlight converters. The use of hybrid solar-fuel systems may allow operating a TPV generator 24 hours a day: at night - a thermal TPV and in the daytime - a solar powered TPV (STPV) system $[5,6]$ with a sunlight concentrator.

In a TPV system there are certain possibilities to improve the efficiency. One of the ways of gaining in the TPV generator efficiency is associated with the use of the energy of the "useless" long-wavelength radiation to make higher the emitter temperature. The low-energy photon recirculation effect can be achieved by introduction of a reflector on the cell back side, for example, by depositing a dielectric cover and a metal film on the back wafer surface.

This paper describes the features of the developed GaSb and Ge cells for TPV applications. In particular, it is shown on the bases of measured photovoltaic parameters of the cells (spectral response, quantum efficiency, open circuit voltage, fill factor) and calculated spectra of the black-body-type photon emitters, that efficiencies as high as $30 \%$ can be achieved in a solar powered TPV system with a concentration ratio of about $10^{4}$ suns, and receiver/emitter photon recycling efficiency of $90 \%$. 


\section{GaSb BASED TPV CELLS}

The following features of the GaSb cells have been under investigation at the presented stage of work:

- reflection coefficient of the sub-bandgap photons for the samples with different doping levels and thicknesses;

- quality evaluation of the GaSb ingots and wafers;

- evaluation of the $\mathrm{Zn}$ profiles after diffusion process;

- estimation of the TPV conversion efficiencies in practical cells.

\section{Reflection Coefficient For Sub-Bandgap Photons}

It had been pointed out that the TPV system efficiency depends to large extent on effective employment of sub-bandgap photons. We have investigated the dependences of the "useless" infrared radiation reflection coefficient for GaSb samples on their free carrier concentration and thickness.

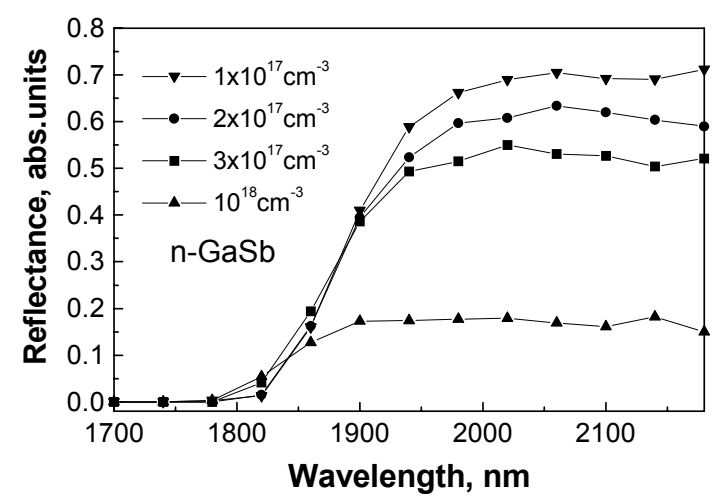

FIGURE 1. Reflectance of the Te-doped n-GaSb samples $(450 \mu \mathrm{m}$ thick) with different doping levels. The samples have the front $\mathrm{ZnS} / \mathrm{MgF}_{2}$ ARC coating and $\mathrm{MgF}_{2} / \mathrm{Au}$ backsurface mirror.

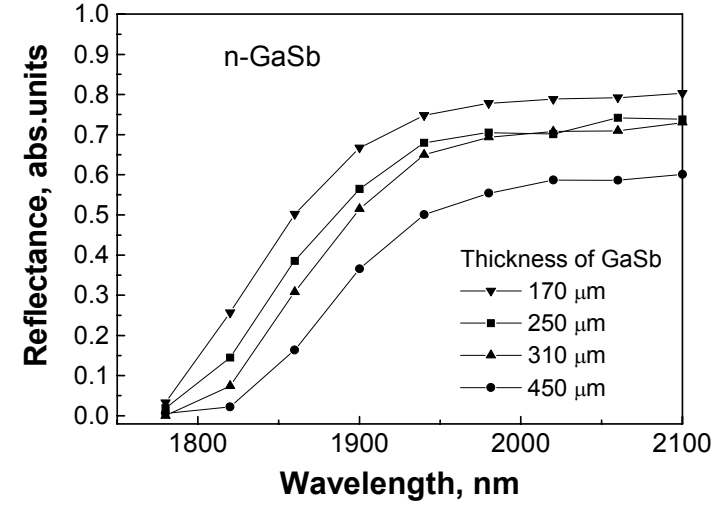

FIGURE 2. Reflectance of the GaSb samples $\left(\mathrm{n}=2 \times 10^{17} \mathrm{~cm}^{-3}\right)$ with different thicknesses. The samples have front $\mathrm{ZnS} / \mathrm{MgF}_{2}$ ARC coating and the $\mathrm{MgF}_{2} / \mathrm{Au}$ back-surface mirror.

In the developed $\mathrm{GaSb}$ cells, the back-surface mirror consists of $\mathrm{MgF}_{2}$ and $\mathrm{Au}$ layers [7]. All samples have $\mathrm{ZnS} / \mathrm{MgF}_{2} \mathrm{ARC}$ coating. Figures 1 and 2 show spectral dependences of reflectance on free carrier concentration and wafer thickness. Figure 3 presents reflectance versus carrier concentration and wafer thickness for $\mathrm{GaSb}$ samples at wavelength $\lambda=2100 \mathrm{~nm}$. As it is seen from these figures maximum reflectance of $80 \%$ at wavelengths longer than $2.0 \mu \mathrm{m}$ may be obtained for GaSb cells $\left(170 \mu \mathrm{m}\right.$ thick $\left.\mathrm{n}=2 \times 10^{17} \mathrm{~cm}^{-3}\right)$. 


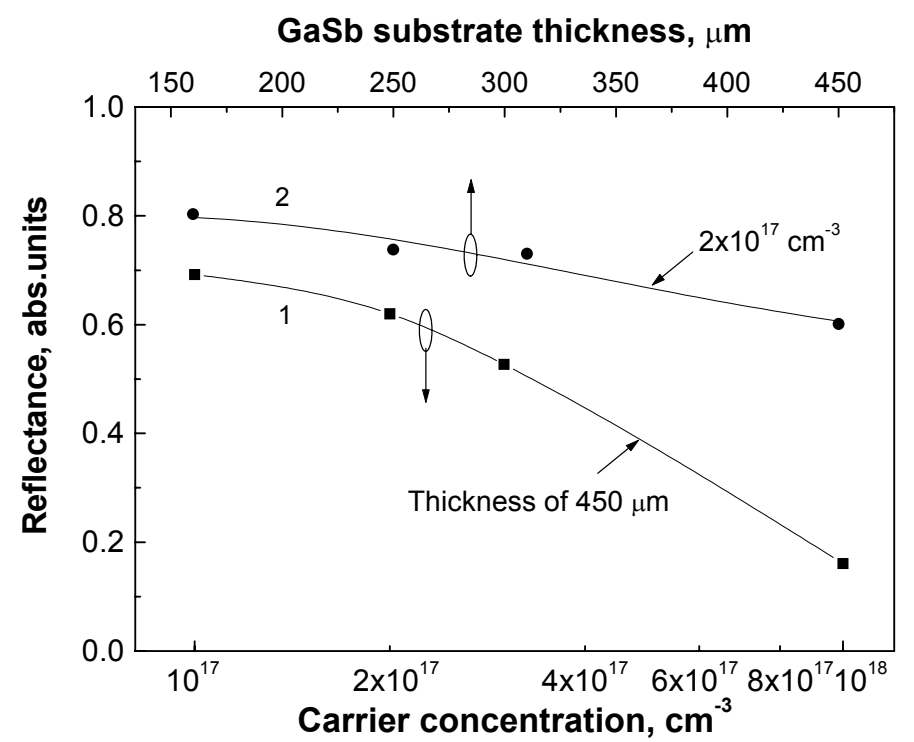

FIGURE 3. Reflectance of $n-G a S b$ samples vs carrier concentration $(450 \mu \mathrm{m}$ thick - curve 1$)$ and thickness $\left(\mathrm{n}=2 \times 10^{17} \mathrm{~cm}^{-3}\right.$ - curve 2$)$ at wavelength $\lambda=2100 \mathrm{~nm}$.

\section{Quality Evaluation Of GaSb Ingots And Wafers}

For GaSb TPV cells a good quality bulk semiconductor material is required to produce high efficient PV converters by Zn diffusion process [7-9].

It has been found (see in Fig. 4, 5) that GaSb ingots grown by the Czochralski technique and doped with tellurium have inhomogeneities due to gradual variation of doping impurity concentration and electron mobility along an ingot. It is seen from the Hall measurements at $77 \mathrm{~K}$, that carrier mobility increases along the ingot in 1.5 - 2 times, whereas for electrically active impurity concentration it is $5-10$ times higher.
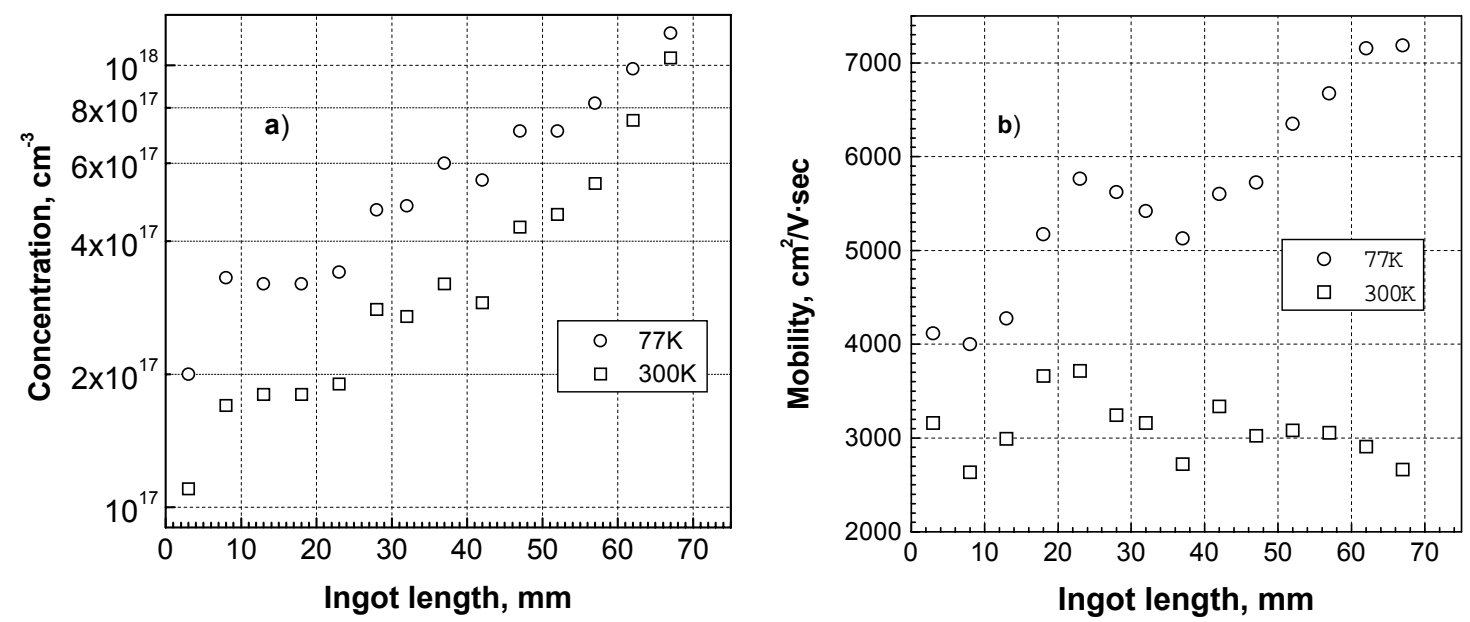

FIGURE 4. Hall measurements of free carrier concentration (a) and mobility (b) along GaSb ingot N96. 


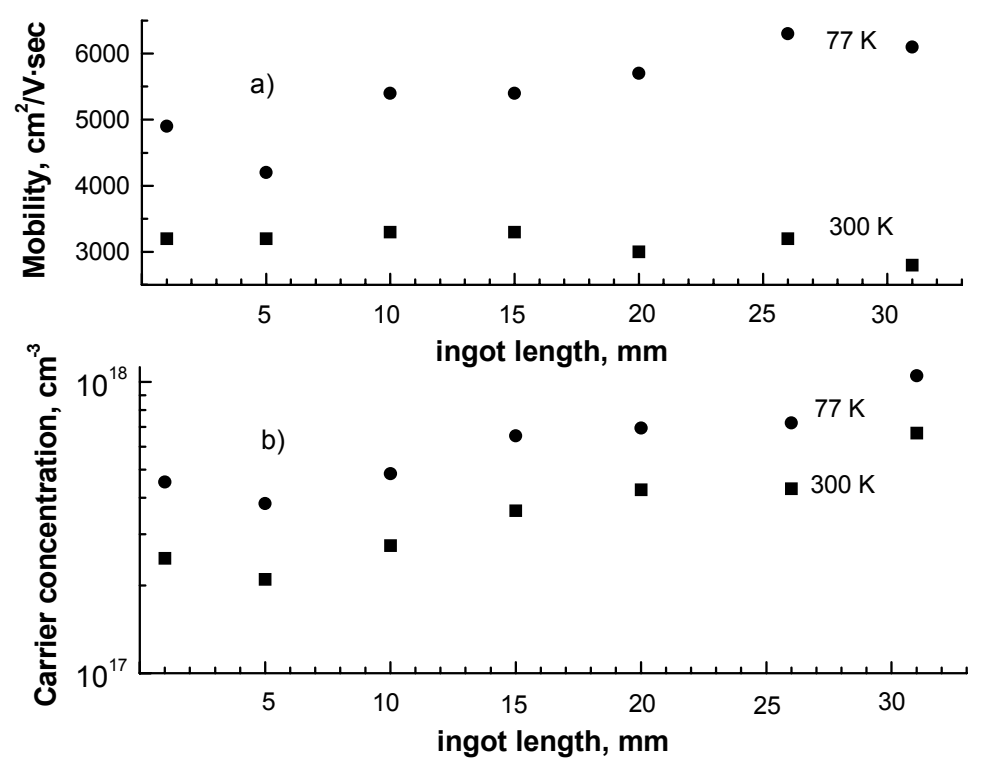

FIGURE 5. Hall measurements of free carrier mobility (a) and concentration (b) along GaSb ingot N93.
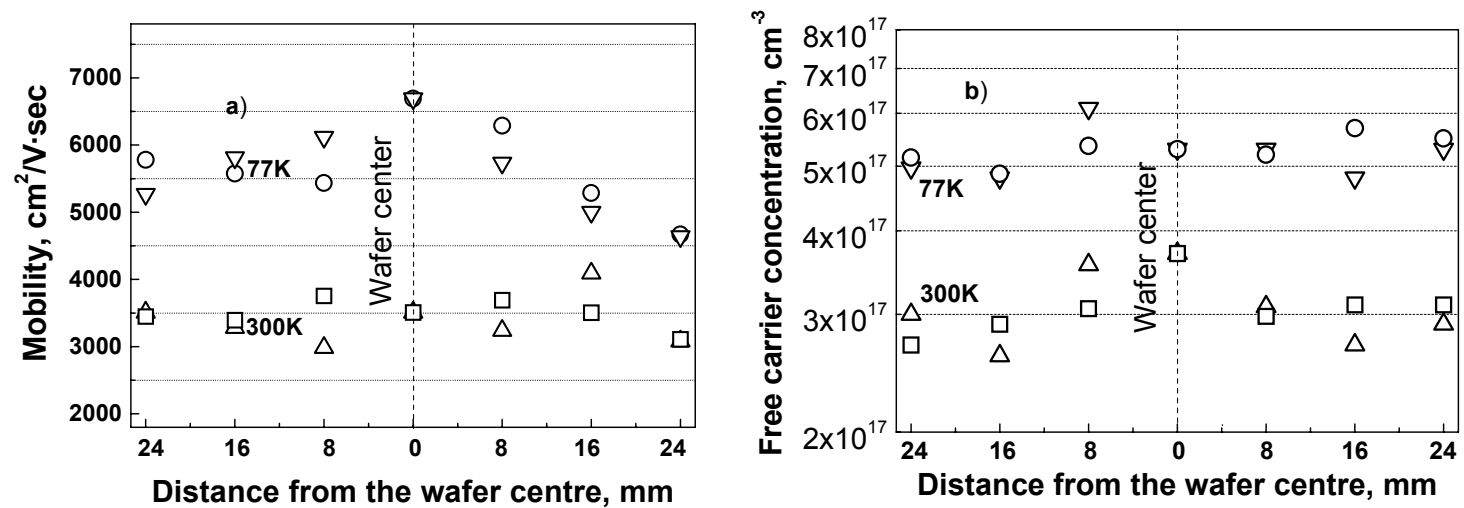

FIGURE 6. Hall measurements of carrier mobility (a) and concentration (b) for GaSb (Te) wafer N36 from ingot N93 in two perpendicular directions.

The concentration gradient of the electrically active impurities along the ingot is, apparently, associated with the rise of Te concentration resulted from enriching the melt with Te during growth period. The carrier mobility value may serve as a measure of existence of additional impurity formations and defects. The mobility in the ingots under investigation increases to the ingot end. Its rise, in spite of the tellurium concentration increase, may be explained, for example, by the uncontrolled impurity content and by formation of clusters in the crystal. The transverse inhomogeneity of the ingot (Fig. 6) resulted from the Te concentration and carrier mobility variations by $20-30 \%$ in the radial direction. The above inhomogeneity values may partly be explained by errors of the Hall measurements, and in total can not influence the main characteristics of TPV cells fabricated with this wafers. 
Thus, near to $80 \%$ of the GaSb ingot may be used for fabrication of wafers for high efficiency TPV cells, but without taking into account the reflection of sub-bandgap photons. And only $30-50 \%$ of the GaSb ingot can be considered as the most suitable for achievement of maximum reflection in GaSb based TPV cells.

\section{Evaluation Of The Zn Profiles}

TPV cells are fabricated by a relatively simple method of zinc diffusion from the gas phase at relatively low temperatures $\left(470-490{ }^{\circ} \mathrm{C}\right)$ in a pseudo-closed box in the hydrogen flow. An increase in the exposure time up to 1 hour allowed us to form $p-n$ junctions laying in the depth of $0.4-0.6 \mu \mathrm{m}$. One of the ways to simplify the technological cycle of formation of an optimum $p$ - $n$ junction relief is $\mathrm{Zn}$ diffusion through semipermeable films deposited on the wafer surface with spacial selection. In this case the base active layer and under-contact regions can be formed during onestage diffusion process.

In the present work we have used the thin layers of the anodic oxide on the $\mathrm{GaSb}$ surface as a special semipermeable mask at $\mathrm{Zn}$ diffusion. Our experiments have shown, that, instead of expected braking of the mass transport, anodic oxidation of the wafer resulted in essential acceleration of $\mathrm{Zn}$ diffusion into bulk GaSb. For example, zinc diffusion from the gas phase through anodic oxide film $0.16 \mu \mathrm{m}$ thick (it was formed at the voltage of $80 \mathrm{~V}$ ) allowed increasing in both $p$ - $n$ junction depth (almost in two times compared to the diffusion into the samples without oxidation) and surface impurity concentration (in $1.5-2$ orders of magnitude). Corresponding profiles of Zn distribution measured by SIMS method are shown in Fig. 7.

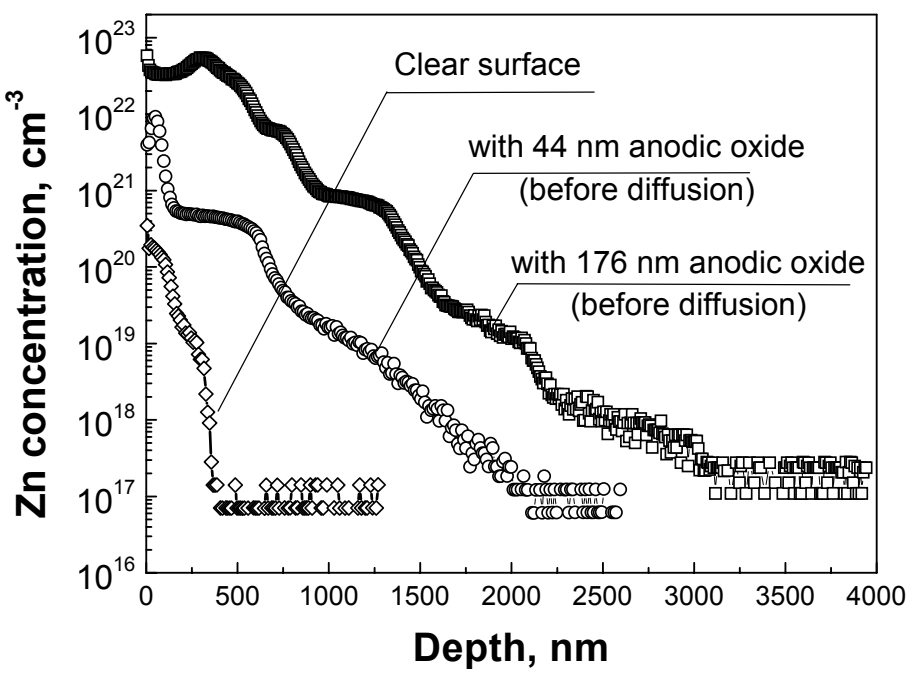

FIGURE 7. Free carrier distribution profiles at $\mathrm{Zn}$ diffusion into $\mathrm{GaSb}$ wafers from the gas phase in the case of clear and oxidized sample surfaces before diffusion.

Therefore, anodic oxide assists the intensive $\mathrm{Zn}$ absorptions from the gas phase. Anodic oxide may be used for local increase in depth of the diffused $p-n$ junction in 
the places intended for contact grid deposition. Being characterized by improved chemical stability after thermal treatment such films are not removed from the GaSb surface.

Introduction of a subcontact layer of anodic oxide after its thermal treatment allows improving contact metal adhesion and even reducing contact resistance.

\section{Estimation Of The TPV Conversion Efficiency}

Back-surface reflection of non-absorbed sub-bandgap photons in a TPV cell allows maximizing the efficiency of a STPV system owing to possible reabsorption of these photons in the emitter. GaSb TPV cell photocurrent densities and efficiencies have been calculated on the basis of measured parameters, such as fill factor, open circuit voltage and photosensitivity spectrum curve of the practical cells developed in the PV Lab of the Ioffe Institute. Corresponding data are plotted in Fig. 8 as the function of a black body IR-emitter temperature for different reflectance values for sub-bandgap photons.

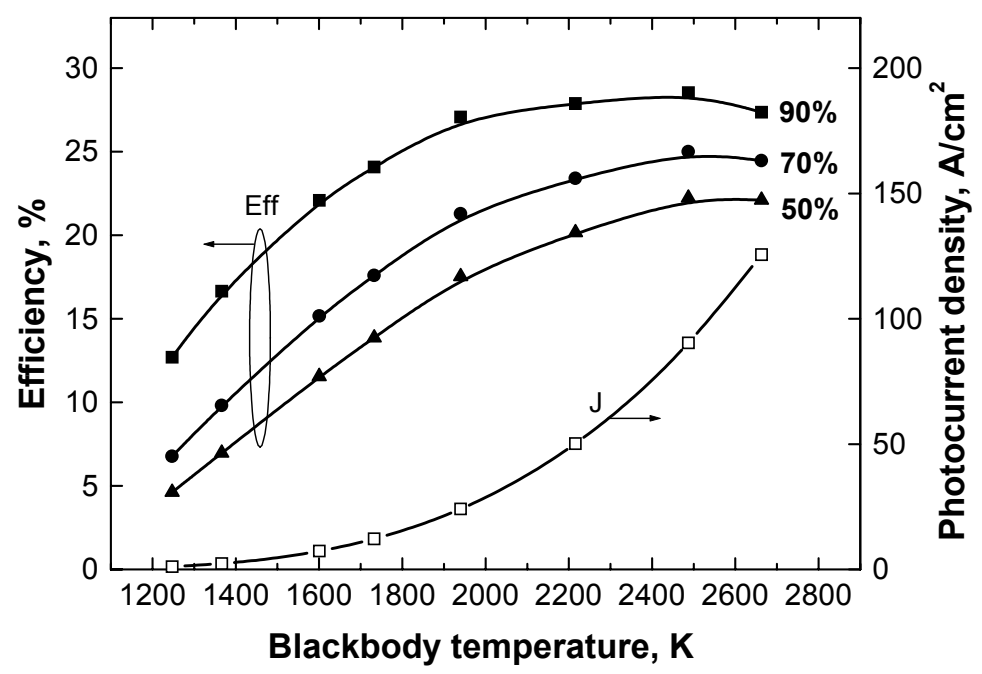

FIGURE 8. GaSb TPV cell photocurrent densities and efficiencies versus black body IR-emitter temperature. Reasonable cell-emitter reflectance values of $50 \%, 70 \%$, and $90 \%$ for sub-bandgap photons are assumed.

Efficiencies as high as $27-28 \%$ are predicted in these cells for the case of $90 \%$ return efficiency and $\mathrm{T}_{\mathrm{BB}}>2000 \mathrm{~K}$.

\section{Ge BASED TPV CELLS}

TPV cells based on GaAs(thin window)/Ge heterostructures have been fabricated by using two approaches. The first of them was combination of metal-organic chemicalvapor deposition of GaAs layer and $\mathrm{Zn}$ diffusion from the gas phase. Fabrication procedure included deposition of a $\mathrm{Si}_{3} \mathrm{~N}_{4}$ dielectric coating, opening the windows in it, and selective diffusion of $\mathrm{Zn}$. Such an approach resulted in a comparatively high 
photocurrent density and open-circuit voltage in the cells, what can be explained by planar growth of a wide-gap GaAs window as thin as $0.1 \mu \mathrm{m}$ and by the fact that the $p-n$ junction does not extend to the opened surface of the cell. In the measurements the open-circuit voltage increased linearly with the photocurrent density up to $0.42-0.43 \mathrm{~V}$ at photocurrent density of $10 \mathrm{~A} / \mathrm{cm}^{2}$. To our knowledge, this is the maximum open-circuit voltage for such Ge based cells measured under similar conditions. Conversion efficiency, being calculated for one-sun photocurrent density of $28.9 \mathrm{~mA} / \mathrm{cm}^{2}$ AM0 spectrum reaches its maximum of $5.5-5.7 \%$ at 200 -fold sunlight concentration [10].

The second approach was the following. The $\mathrm{p}-\mathrm{GaAs} / \mathrm{p}-\mathrm{Ge} / \mathrm{n}-\mathrm{Ge}$ heterostructures with a thin $(0.1 \mu \mathrm{m}) \mathrm{GaAs}$ layer were grown by low-temperature $\left(380{ }^{0} \mathrm{C}\right)$ liquid-phase epitaxy. Additional Zn-diffusion process into the p-GaAs-n-Ge heterostructure was necessary in this case. The advantage of this technology compared to MOCVD is avoiding the undesirable diffusion of $\mathrm{Ga}$ and As atoms from vapour phase into Ge owing to much lower growth temperature in LPE process.

Reflectance measurements for Ge samples with different types of back surface mirrors and different doping levels of bulk material have been carried out. The results are presented in Fig. 9, 10. Reflectance above 90\% has been achieved in Ge samples $300 \mu \mathrm{m}$ thick with doping level $\mathrm{n}=10^{17} \mathrm{~cm}^{-3}$.

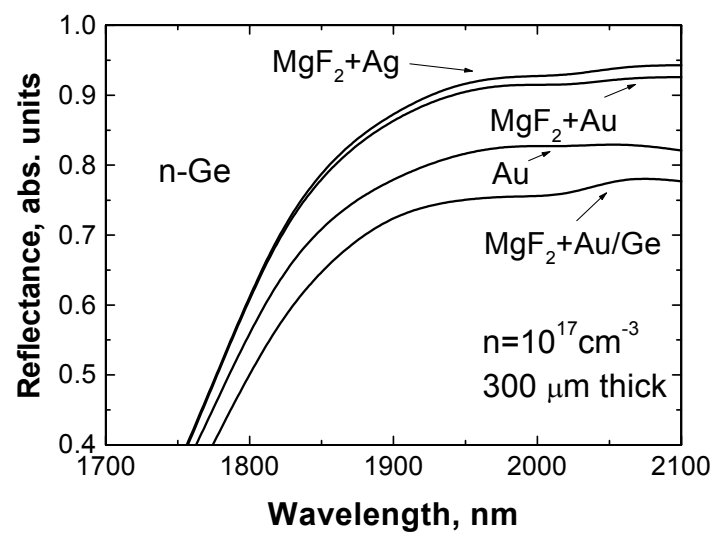

FIGURE 9. Reflectance vs. wavelength for n-Ge samples with different back surface mirrors. The samples have front $\mathrm{ZnS} / \mathrm{MgF}_{2}$ ARC coating.

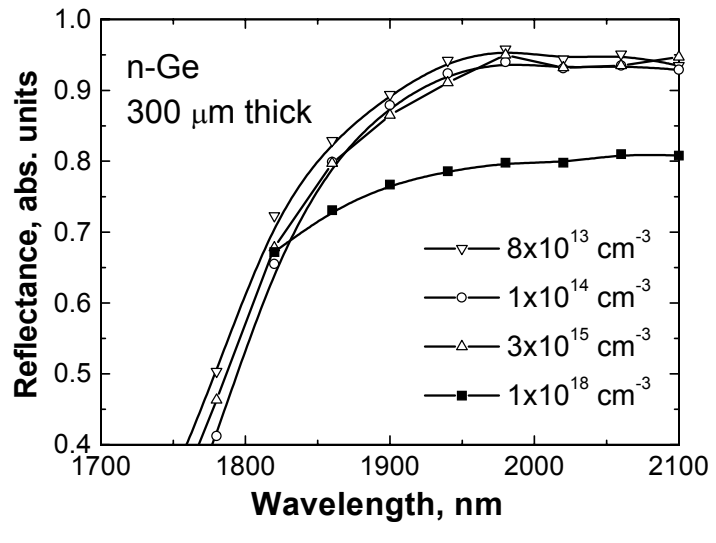

FIGURE 10. Reflectance vs. wavelength for n-Ge samples with different doping levels. Samples have $\mathrm{ZnS} / \mathrm{MgF}_{2}$ ARC coating and $\mathrm{MgF}_{2} / \mathrm{Au}$ back surface mirror.

Figure 11 demonstrates dependences of LPE grown GaAs/Ge TPV cell efficiencies, calculated on the basis of practically measured photovoltaic parameters in the same way, as it was done for GaSb cells. Efficiency of $16 \%$ is predicted in these cells under black-body irradiation $(1700-2100 \mathrm{~K})$ assuming 100\% reflectance for sub-bandgap photons.

Comparison of the results presented in Fig. 8 and Fig. 11 shows that efficiencies in $\mathrm{Ge}$ cells are lower than in GaSb cells. However, the developed GaAs/Ge cells have prospects for application in solar TPV systems taking into account the lower price of 
Ge wafers and possibility to obtain a higher reflectance (Fig. 9, 10) for these cells in comparison with GaSb cells (Fig. 1-3).

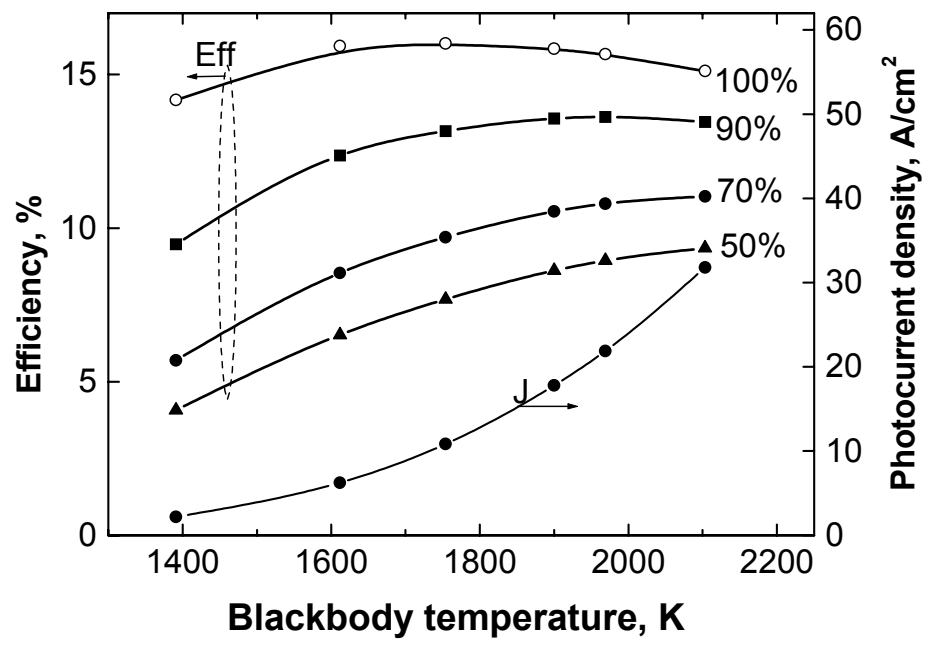

FIGURE 11. LPE grown GaAs/Ge TPV cell efficiencies as a function of a black-body emitter temperature. Reasonable cell-emitter reflectance values of $50 \%, 70 \%, 90 \%$ and $100 \%$ reflection of subbandgap photons from the cell to the emitter is assumed.

\section{CONCLUSIONS}

Reflectance measurements for GaSb and Ge based samples with different types of back surface mirrors in dependence on bulk doping level ( $\mathrm{GaSb}, \mathrm{Ge})$ and thickness (GaSb) have been carried out. Maximum measured reflectance is about $80 \%$ for the GaSb samples $170 \mu \mathrm{m}$ thick with the doping level of $2 \times 10^{17} \mathrm{~cm}^{-3}$. More than $90 \%$ reflectance has been achieved for Ge samples $300 \mu \mathrm{m}$ thick with free carrier concentration of $10^{17} \mathrm{~cm}^{-3}$.

The TPV efficiencies of 27-28\% have been achieved in GaSb cells fabricated by the optimised $\mathrm{Zn}$ diffusion method. Cell efficiencies have been calculated on the basis of PV parameters measured in practical cells. Similar calculations for developed Ge based cells with GaAs window layer gave lower efficiencies (no more, than 16\%).

\section{ACKNOWLEDGEMENTS}

The authors wish to thank Zh.Alferov and A.Luque for helpful discussions and to express our gratitude to N.H.Timoshina and A.I.Dement'eva for their contribution to this work.

This work has been supported by the European Commission through the funding of the project FULLSPECTRUM (Ref. N: SES6-2003-502620). 


\section{REFERENCES}

1. P. A. Davies, A. Luque, "Solar thermophotovoltaics: Brief review and a new look", Solar Energy Materials \& Solar Cells, 33, 11-22 (1994).

2. V. Badescu, "Thermodinamic theory of thermophotovoltaic solar energy conversion", J.Appl. Phys., 90, 6476-6486 (2001).

3. K. W. Stone, N. S. Fatemi, L. Garverick, "Operation and component testing of a solar thermophotovoltaic power system", Proc. of $25^{\text {th }}$ IEEE PVSC, Washington, DC, 1996, 1421-1424.

4. H. Yugami, H. Sai, K. Nakamura, N. Nakagama, H. Ohtsubo, "Solar thermophotovoltaic using $\mathrm{Al}_{2} \mathrm{O}_{3} / \mathrm{Er}_{3} \mathrm{Al}_{5} \mathrm{O}_{12}$ eutectic composite selective emitter" Proc. $28^{\text {th }}$ IEEE PVSC, Anchorage, 2000, pp. 1214-1217.

5. V. D. Rumyantsev， V. P. Khvostikov， O. A. Khvostikova， P. Y. Gazaryan, N. A. Sadchikov, A. S. Vlasov, E. A. Ionova, V. M. Andreev "Structural Features of a Solar TPV Systems", $6^{\text {th }}$ Conference on Thermophotovoltaic Generation of Electricity, Freiburg, 2004 (in this book).

6. V. M. Andreev, V. P. Khvostikov, O. A. Khvostikova, V. D. Rumyantsev, P. Y. Gazaryan, A. S. Vlasov, "Solar Thermophotovoltaic Converters: Efficiency Potentialities", $\sigma^{\text {th }}$ Conference on Thermophotovoltaic Generation of Electricity, Freiburg, 2004 (in this book).

7. V. M. Andreev, V. P. Khvostikov, O. A. Khvostikova, E. V. Oliva, V. D. Rumyantsev, M. Z. Shvarts, "Thermophotovoltaic Cells with Sub-bandgap Photon Recirculation", Proc. of 17th European Photovoltaic Solar Energy Conf., Munich, 2001, pp. 219-222.

8. A. W. Bett, S. Keser, O. V. Sulima "Study of Zn Diffusion into GaSb from the Vapour and Liquid Phase", J. of Crystal Growth 181, pp. 9-16 (1997).

9. G. Stollwerck, O. V. Sulima, A. W. Bett, "Characterization and Simulation of GaSb DeviceRelated Properties", IEEE Transactions on Electron Devices 47 (2), pp. 448-457 (2000).

10. V. Andreev, V. Khvostikov, O. Khvostikova, N. Kaluzhniy, E. Oliva, V. Rumyantsev, S. Titkov, M. Shvarts, "Low-Bandgap PV and Thermophotovoltaic Cells", 3rd World Conference on Photovoltaic Energy Conversion, Osaka (2003). 